\title{
Evans blue removal from wastewater by rarasaponin-bentonite
}

\section{K. Chandra, Y.-H. Ju, A. Ayucitra \& S. Ismadji}

International Journal of Environmental Science and Technology

ISSN 1735-1472

Volume 10

Number 2

Int. J. Environ. Sci. Technol. (2013)

10:359-370

DOI 10.1007/s13762-012-0114-y

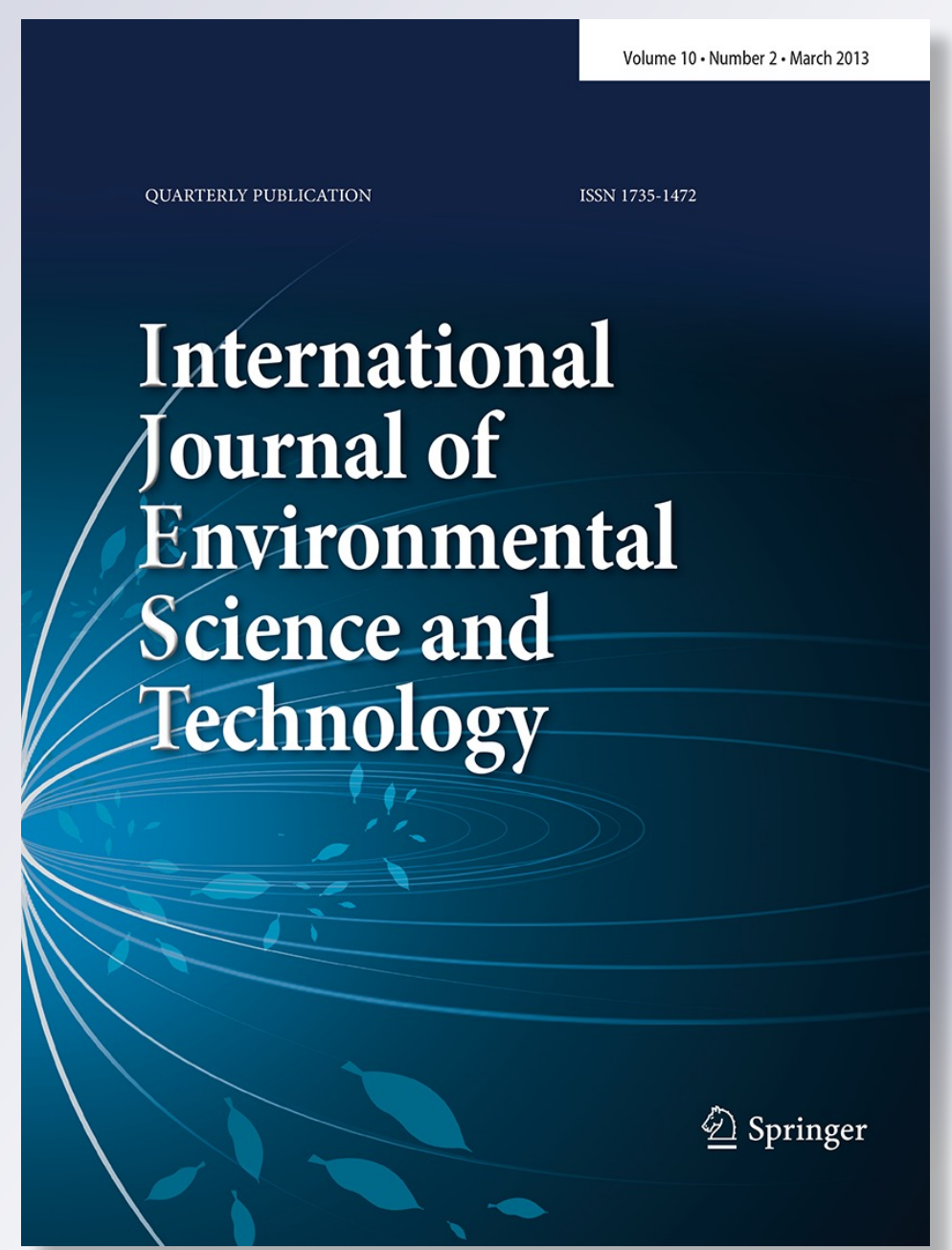

望 Springer 
Your article is protected by copyright and all rights are held exclusively by CEERS, IAU. This e-offprint is for personal use only and shall not be self-archived in electronic repositories. If you wish to self-archive your work, please use the accepted author's version for posting to your own website or your institution's repository. You may further deposit the accepted author's version on a funder's repository at a funder's request, provided it is not made publicly available until 12 months after publication. 


\title{
Evans blue removal from wastewater by rarasaponin-bentonite
}

\author{
I. K. Chandra $\cdot$ Y.-H. Ju • A. Ayucitra • \\ S. Ismadji
}

Received: 2 November 2011/Revised: 3 January 2012/ Accepted: 25 September 2012/Published online: 31 October 2012

(c) CEERS, IAU 2012

\begin{abstract}
The feasibility of natural bentonite and rarasaponin-bentonite for Evans blue removal from aqueous solution was studied. Rarasaponin is a natural surfactant obtained from Sapindus rarak DC was used as modifying agent for natural bentonite modification. Adsorption experiments were conducted in a batch system at various temperatures. Several temperature-dependent isotherm models (Langmuir, Freundlich, Sips and Toth) were used to represent equilibrium data. It was found that Toth model represents the adsorption equilibrium data better than other models. Kinetic data were best represented by the pseudofirst order model. The controlling mechanism of the adsorption of Evans blue onto natural bentonite and rarasaponin-bentonite was physical adsorption.
\end{abstract}

Keywords Bentonite - Modification - Adsorption · Rarasaponin

\section{Introduction}

Many methods have been developed for the purpose of separation or removal of contaminants from industrial wastewater. Among them, adsorption process is still continuously used for sequestering hazardous chemicals from wastewater. In the adsorption process, the correct choice of

I. K. Chandra - Y.-H. Ju

Department of Chemical Engineering, National Taiwan

University of Science and Technology, 43, sec. 4 Keelung Rd.,

Taipei 106, Taiwan

I. K. Chandra $\cdot$ A. Ayucitra $\cdot$ S. Ismadji $(\bowtie)$

Department of Chemical Engineering, Widya Mandala Surabaya Catholic University, Kalijudan 37, Surabaya 60114, Indonesia

e-mail: suryadiismadji@yahoo.com solid adsorbent is the main key for the success of the process. Activated carbons have been known as superior adsorbents for industrial water or wastewater treatment. However, the main obstacle in using these adsorbents is its price, the commercial activated carbons with high adsorption capacities are expensive, and in the view of economics, the use of these adsorbents for wastewater treatment will significantly increase the production cost of the industry.

The search of alternative low-cost adsorbents for wastewater treatment purpose has been begun several decades ago. Mostly the studies focus on biomass-based adsorbents, and some of them focus on clay minerals. Many review papers have summarized the studies on the utilization of biomass-based materials as the low-cost adsorbents for the removal of various organics and heavy metals from simulated wastewater (Wang and Chen 2006; Volesky 2007; Arief et al. 2008; Lesmana et al. 2009; Febrianto et al. 2009). Indeed, the use of these low-cost adsorbents will reduce the production and operational cost, however for industrial application purpose, the availability of these kinds of adsorbents in large quantity and with constant adsorption capacity is still questionable.

Currently, the use of clay minerals for industrial wastewater treatment applications is strongly recommended due to their local availability, technical feasibility, engineering applications, and cost effectiveness. Among the available clay materials, natural bentonite is the most suitable as the adsorbent for adsorption of pollutants from industrial wastewater. However, because of the hydrophilicity induced by the exchangeable metal cations, natural bentonite usually is not effective in adsorbing organic compounds. The surface of natural bentonite can be changed from hydrophilic to hydrophobic or organophilic by organo-functional molecules such as surface hydroxyl 
groups, Lewis and Bronsted acidic sites, etc., by grafting organic groups on the clay surface. For this purpose, the most commonly used chemicals as modifying agents are surfactants (Juang et al. 2002; Lee et al. 2002; Khenifi et al. 2007; Koswojo et al. 2010; Rahardjo et al. 2011). However, most of the surfactants in previous studies are synthetics, which often create serious problem to environment and commonly need expensive waste treatment system for excess or unused surfactants.

In relation with environment protection, the study of modification clay using surfactants should be directed towards the use of natural surfactants as modifying agents. In this study, we employed a natural surfactant called as rarasaponin obtained from the extraction of flesh fruit of Sapindus rarak DC as modifying agent for rarasaponinbentonite preparation. The natural bentonite used in this study was obtained from a natural bentonite mining located near the border of Pacitan and Ponorogo, East Java, Indonesia. The adsorption capability of rarasaponin-bentonite was tested by adsorption of dye from synthetic wastewater solution. Evans blue was taken as the dye model. This dye is chemically and photolytically stable, also highly persistent in natural environment due to the presence of a chromophore group in its molecular structure. It means that the release of this compound in the environment may spread the potential danger of bioaccumulation that may eventually affect human by transport through the food chain (Zee 2002). The adsorption isotherm data obtained in this study were correlated by Langmuir, Freundlich, Sips, and Toth models with its temperaturedependent forms.

\section{Materials and method}

Materials

Dried Sapindus rarak fruit was obtained from Klaten, Central Java, Indonesia. The fruit flesh was separated from the seed and then extracted using deionized water (flesh:solvent $=$ 1:10) at $80^{\circ} \mathrm{C}$ for $4 \mathrm{~h}$. The filtrate was separated from the mixture using filter paper. Subsequently the filtrate was evaporated using Buchi RE 121 rotary evaporator and the concentrate was moved into the plastic tubes. The concentrate then dried using Labconco freeze dryer for $24 \mathrm{~h}$. The dried rarasaponin was ground and sieved using Retsch Haan screen and the rarasaponin powder with the size of 100/120 mesh was collected and kept in desiccators for further use. The chemical functional groups of rarasaponin powder were determined by Fourier Transform Infrared Spectroscopy (FTIR) and the result is given in Fig. 1.

The natural bentonite was obtained from a natural bentonite mining located near the border of Pacitan and Ponorogo, East Java, Indonesia. Prior to use, the natural bentonite was purified to remove organic impurities. The natural bentonite was treated by contacting with $30 \%$ hydrogen peroxide solution for $24 \mathrm{~h}$. The excess of hydrogen peroxide was then removed by heating the mixture at around $100{ }^{\circ} \mathrm{C}$ for $1 \mathrm{~h}$. The treated natural bentonite was then separated from the mixture by centrifugation, and dried at $110{ }^{\circ} \mathrm{C}$ for $24 \mathrm{~h}$. Subsequently, the dried natural bentonite was crushed using Janke \& Kunkel micro hammer mill and sieved using Retsch Haan screen. The chemical composition of the natural bentonite-powder was analyzed using Rigaku ZSX100e X-Ray Fluorescence. The cation exchange capacity (CEC) of this natural bentonite was analyzed using ASTM C837-99 procedure (63.95 meq/100 g of clay).

The diazo dye, Evans blue $\left(\mathrm{C}_{34} \mathrm{H}_{24} \mathrm{~N}_{6} \mathrm{Na}_{4} \mathrm{O}_{14} \mathrm{~S}_{4}\right.$, CAS No. 46160) was purchased from Fluka. Analytical grade of hydrogen peroxide was purchased from Sigma Aldrich. These compounds were used directly without any further purification.

Rarasaponin-bentonite preparation

Rarasaponin obtained from previous treatment was mixed with natural bentonite powder and dispersed in deionized
Fig. 1 FTIR spectra for rarasaponin

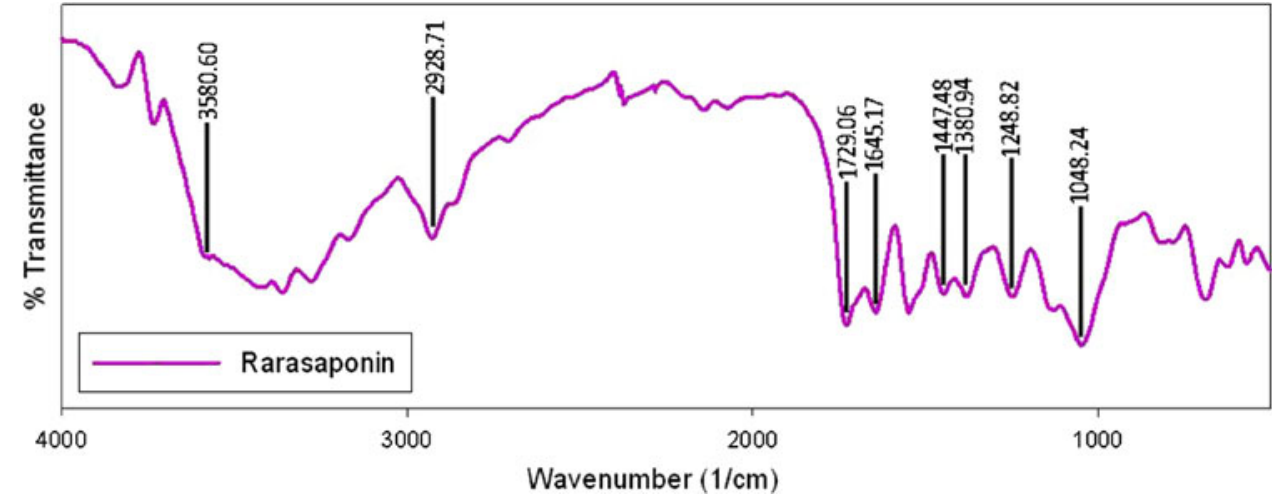


water and then stirred at $500 \mathrm{rpm}$ for $2 \mathrm{~h}$. The ratio of rarasaponin, natural bentonite, and deionized water was $0.5 \mathrm{~g}, 5 \mathrm{~g}$, and $25 \mathrm{~mL}$, respectively. Then, the mixture was put into Inextron microwave oven, and heated for $5 \mathrm{~min}$ at $700 \mathrm{~W}$. The resultant (rarasaponin-bentonite) was repeatedly washed using distilled water to remove excess rarasaponin. The rarasaponin-bentonite was dried at $100{ }^{\circ} \mathrm{C}$ for $24 \mathrm{~h}$. The dried rarasaponin-bentonite was then pulverized until its particle size was around 100/120 mesh.

\section{Characterization of adsorbents}

The natural bentonite and rarasaponin-bentonite was characterized using X-Ray Diffraction (XRD), FTIR, and nitrogen sorption methods. The X-Ray Diffraction analysis was carried out using a Rigaku Miniflex Goniometer instrument using $\mathrm{Cu} \alpha \mathrm{K}$ radiation at $30 \mathrm{kV}$ and $15 \mathrm{~mA}$ with a step size of $0.01^{\circ}$. The FTIR qualitative analysis was performed in FTIR Shimadzu 8400s. The method employed for FTIR analysis was $\mathrm{KBr}$ technique. To identify the pore structure of both natural bentonite and rarasaponin-bentonite, nitrogen sorption analysis of both samples was carried out in Quadrasorb SI at boiling point of nitrogen gas $\left(-196^{\circ} \mathrm{C}\right)$. Before the gas adsorption measurement, the samples were degassed at $150{ }^{\circ} \mathrm{C}$ under vacuum condition for $24 \mathrm{~h}$. The nitrogen sorption measurements were conducted at relative pressure $\left(p / p_{\mathrm{o}}\right)$ in the range of $0.001-0.998$.

\section{Adsorption procedure}

The adsorption isotherm study was performed in batch mode at various temperatures $\left(30,45\right.$, and $\left.60{ }^{\circ} \mathrm{C}\right)$. The known amount of adsorbent was added in a series of Erlenmeyer flasks containing $100 \mathrm{~mL}$ of $0.52 \mathrm{mmol} / \mathrm{L}$ Evans blue solution. These flasks were then moved into Memmert type WB-14 thermostatic shaker bath and shaken at $100 \mathrm{rpm}$. The temperature of the thermostatic shaker bath was adjusted to a desired temperature. When equilibrium condition was reached, the adsorbent was separated from the solution by centrifugation (Hettich Zentrifugen EBA-20). The initial and equilibrium concentration of Evans blue in the solution was determined by Shimadzu UV/VIS-1700 Pharmaspec spectrophotometer at its maximum wavelength. The amount of Evans blue adsorbed by the adsorbent was calculated by the following equation:

$q_{\mathrm{e}}=\left(\frac{c_{\mathrm{o}}-c_{\mathrm{e}}}{m} V\right)$

where $q_{\mathrm{e}}$ is the amount of Evans blue adsorbed (mmol/g), $m$ is the mass of adsorbent $(\mathrm{g})$, while $V$ is the volume of solution (L), and $C_{\mathrm{o}}$ and $C_{\mathrm{e}}$ are initial and equilibrium concentration $(\mathrm{mmol} / \mathrm{L})$, respectively.

For the kinetic study, the experiments were also conducted isothermally in three different temperatures $(30,45$, and $\left.60{ }^{\circ} \mathrm{C}\right)$. A series of Erlenmeyer each containing 100-ml Evans blue solution was mixed with $1 \mathrm{~g}$ of adsorbent. The flasks containing the mixtures were then shaken at $100 \mathrm{rpm}$ in Memmert type WB-14 thermostatic shaker bath at certain temperature. At certain interval of time, one of the flasks was taken from the thermostatic shaker bath, and the mixture was separated using centrifugation. The concentration of remaining Evans blue solution was determined using spectrophotometer, and the amount of Evans blue adsorbed at time interval $t$ was determined by the following equation

$\mathrm{q}_{t}=\frac{\left(c_{\mathrm{o}}-c_{t}\right)}{m} V$

where $C_{t}$ is the concentration of Evans blue at time interval $t$.

\section{Theory}

Adsorption is regarded as practical separation method for purification or bulk separation of different kind of industrial products. In the field of environmental pollution control, the adsorption process also plays significant role, especially in the wastewater treatment system. Adsorption equilibrium and kinetic are the most important aspects for the understanding of engineering design methods of adsorption system. The adsorption equilibrium is the most fundamental property and can be correlated through mathematical formulation called as adsorption isotherm. Different kinds of adsorption isotherm models have been developed and proposed. Some of them are based on simplified physical phenomena of adsorption, while others are purely empirical with two or three empirical parameters.

The adsorption of adsorbate onto an adsorbent is affected by temperature, and in most cases the comparisons between the adsorption equilibrium obtained from the experiments and adsorption isotherm model have been made at single temperature. Since the adsorption equilibria are temperature dependent, it is important to include the temperature dependent forms of adsorption models in correlating the experimental data. In this study we used the temperature dependent forms of Langmuir, Freundlich, Sips, and Toth models. A brief description of the temperature dependent forms of these models are given in this paper, and for complete discussion of these temperature dependent forms the reader can refer to Do (1998). 
Langmuir isotherm

Langmuir (1918) proposed a theory of adsorption on a flat surface (Do 1998). The theory is based on a kinetic principle, that is the rate of adsorption is equal to the rate of desorption from the surface. The famous Langmuir model is

$q_{\mathrm{e}}=q_{\max }\left(\frac{K_{\mathrm{L}} C_{\mathrm{e}}}{1+K_{\mathrm{L}} C_{\mathrm{e}}}\right)$

where $C_{\mathrm{e}}$ is the equilibrium concentration of the solution $(\mathrm{mmol} / \mathrm{L})$ and $K_{\mathrm{L}}$ is a Langmuir affinity constant $(\mathrm{L} / \mathrm{mmol})$, $q_{\max }(\mathrm{mmol} / \mathrm{g})$ is the adsorption capacity of the adsorbate, while $q_{\mathrm{e}}(\mathrm{mmol} / \mathrm{g})$ is the amount of dye adsorbed per unit mass of adsorbent.

The temperature dependent forms of the Langmuir isotherm parameters $\left(q_{\max }\right.$ and $\left.K_{\mathrm{L}}\right)$ can be written in the following forms:

$q_{\max }=q_{\max }^{0} \exp \left[\delta\left(T_{0}-T_{1}\right)\right]$

$K_{\mathrm{L}}=K_{\mathrm{L}}^{0} \exp \left[\frac{E}{\mathrm{RT}_{0}}\right]$

where $q_{\max }$ is the adsorption capacity at reference temperature $T_{0}$, while the temperature coefficient of the expansion of adsorbate is $\delta$. The parameters $E$ and $K_{\mathrm{L}}^{\mathrm{o}}$ are the heat of adsorption and the Langmuir affinity constant at reference temperature $\left(T_{0}\right)$, respectively.

Freundlich isotherm

As one of the earliest empirical equations and most widely used isotherm model to describe the adsorption equilibrium data, Freundlich model (1932) has the form

$q_{\mathrm{e}}=K_{\mathrm{F}} C_{\mathrm{e}}^{1 / n}$

where $K_{\mathrm{F}}(\mathrm{mmol} / \mathrm{g})(\mathrm{L} / \mathrm{mmol})^{-n}$ is the measure of Freundlich adsorption capacity and $1 / n$ is adsorption intensity.

Parameters $K_{\mathrm{F}}$ and $n$ are the complex forms of dependent temperature, and one should not extrapolate them outside their range of validity (Do 1998). The temperature dependent forms of Freundlich parameters $\left(K_{\mathrm{F}}\right.$ and $\left.n\right)$ can be written as

$K_{\mathrm{F}}=K_{\mathrm{F}}^{0} \exp \left[\frac{-\alpha \mathrm{RT}}{A_{0}}\right]$

$\frac{1}{n}=\frac{R T}{A_{0}}$

The parameter $K_{\mathrm{F}}^{\mathrm{o}}$ indicates the Freundlich adsorption capacity at reference temperature $\left(T_{0}\right)$, while $\alpha$ and $A_{0}$ are the characteristics of adsorption potential and constant parameter of Clapeyron, respectively.
Sips isotherm

Sips equation which also known as the Langmuir-Freundlich equation has the form

$q_{\mathrm{e}}=q_{\max }\left[\frac{\left(K_{\mathrm{s}} C_{\mathrm{e}}\right)^{1 / n}}{1+\left(K_{\mathrm{s}} C_{\mathrm{e}}\right)^{1 / n}}\right]$

where $K_{\mathrm{S}}(\mathrm{mmol} / \mathrm{L})^{1 / n}$ is Sips affinity constant and parameter $n$ is regarded as the parameter characterising the system heterogeneity. When $n$ is unity, the Sips equation reduces to Langmuir, which is suitable for ideal surfaces (Do 1998).

Parameters $K_{\mathrm{S}}$ and $n$ have temperature dependent forms as follow:

$K_{\mathrm{S}}=K_{\mathrm{S}}^{0} \exp \left[\frac{E}{\mathrm{RT}_{0}}\left(1-\frac{T_{0}}{T}\right)\right]$

$n=\frac{1}{\frac{1}{n_{0}}+n\left(1-\frac{T_{0}}{T}\right)}$

The parameters $K_{\mathrm{S}}^{0}$ and $n_{0}$ are Sips parameters at the reference temperature $\left(T_{0}\right)$, while $\eta$ is a constant parameter.

Toth isotherm

While Freundlich equation is not valid at low and high concentration and Sips equation also has limitation at low end concentration range, Toth equation gives satisfactory results especially for Henry law type behaviour (Do 1998). This equation is suitable for sub-monolayer coverage system and has the following form:

$q_{\mathrm{e}}=\frac{q_{\mathrm{max}} C_{\mathrm{e}}}{\left(K_{\mathrm{Th}} \times C_{\mathrm{e}}^{t}\right)^{1 / t}}$

Parameter $t$ that is usually less than unity and has the same physical meaning with Sips parameter $(n)$ indicates the system heterogeneity. When its value deviates further away from unity, the system becomes more heterogeneous (Do 1998). Parameter $K_{\mathrm{T}}(\mathrm{mmol} / \mathrm{L})^{t}$ is Toth affinity constant.

Toth equation also has temperature dependent forms, which is useful to describe adsorption equilibrium data at various temperatures. The temperature dependent forms of Toth model can be written as

$K_{\mathrm{Th}}=K_{\mathrm{Th}}^{0} \exp \left[\frac{E}{\mathrm{RT}_{0}}\left(\frac{T_{0}}{T}-1\right)\right]$

$t=t_{0}+\eta\left(1-\frac{T_{0}}{T}\right)$

The affinity coefficient $\left(K_{\mathrm{TH}}^{0}\right)$ and parameter $t_{0}$ are at the reference temperature $\left(T_{0}\right)$, while $\eta$ is a constant parameter. 
Theory of adsorption kinetics

To properly design an adsorption system, additional information beside the adsorption equilibria is needed. This crucial information is the adsorption kinetic. The rate of pollutants adsorbed into the adsorbent is one of the crucial factors which influence the effectiveness of the sorption process (Plazinski et al. 2009). Many mathematical models have been proposed, but pseudofirst order and pseudo-second order are still extensively applied to describe the kinetics of sorption in solid/ solution systems.

\section{Pseudo-first order}

This model proposed by Lagergren in nineteenth century (Lagergren 1898), and the pseudo-first order equation became popular to describe the rate of sorption in the liquid-phase systems. The differential form of the pseudofirst model is as follows:

$\frac{\mathrm{d} q(t)}{\mathrm{d} t}=K_{1}\left(q_{\mathrm{e}}-q(t)\right)$

The integration form of Eq. (15) can be written as follows:

$q(t)=q_{\mathrm{e}}\left(1-\exp \left(-k_{1} t\right)\right)$

Pseudo-second order

Another model which is widely used for interpretation of adsorption kinetic data is the pseudo-second order. The pseudo-second order kinetic is usually associated with the situation when the rate of direct adsorption/desorption process controls the overall sorption kinetics (Plazinski et al. 2009). This model was first developed by Blanchard et al. (1984) and the linearized form of this model proposed by Ho (1995). The mathematical form of the pseudo-second order model is

$\frac{\mathrm{d} q(t)}{\mathrm{d} t}=k_{2}\left(q_{e}-q(t)\right)^{2}$

By integrating of Eq. (17) the final form of the pseudosecond order is

$q(t)=q_{\mathrm{e}}\left(\frac{q_{\mathrm{e}} k_{2} t}{1+q_{\mathrm{e}} k_{2} t}\right)$

Both pseudo-first and pseudo-second order equations have parameter time constant $k$ ( $\mathrm{g} / \mathrm{mmol} \mathrm{min})$ to describe the rate constant of adsorption, the symbol $q_{t}(\mathrm{mmol} / \mathrm{g})$ represents the amount of adsorbate on the surface of the adsorbent at any time, $t$ (Plazinski et al. 2009).
Table 1 The FTIR spectra of rarasaponin

\begin{tabular}{ll}
\hline Functional group & Wavenumber $(1 / \mathrm{cm})$ \\
\hline O-H stretch, free hydroxyl & $3,580.60$ \\
$\mathrm{C}-\mathrm{H}$ stretch & $2,928.71$ \\
$\mathrm{C}=\mathrm{O}$ stretch (ester carbonyls group) & $1,729.06$ \\
$\mathrm{C}=\mathrm{C}$ strecth (olefin group) & $1,645.17$ \\
$\mathrm{C}-\mathrm{CH}_{3}$ bend & $1,447.48$ \\
$\mathrm{C}-\mathrm{H}$ bend bonded with hydroxyl group & $1,380.94$ \\
$\mathrm{C}-\mathrm{O}$ stretch (carbonyls group) & $1,248.82$ \\
$\mathrm{C}=\mathrm{C}$ stretch (ethers group) & $1,048.24$ \\
\hline
\end{tabular}

\section{Results and discussion}

Identification of rarasaponin

The FTIR spectrum of rarasaponin obtained from extraction of Sapindus rarak is given in Fig. 1; Table 1. The hydroxyl group of rarasaponin was shown at wavenumber of $3,580.60 \mathrm{~cm}^{-1}$, while the wavenumber $1,729.06 \mathrm{~cm}^{-1}$ indicates ester carbonyl group. Olefin group and ethers group were identified at wavenumber $1,645.17$ and $1,048.24 \mathrm{~cm}^{-1}$, respectively. These functional groups indicates that the structure of rarasaponin belongs to oligosaccharide (Asao et al. 2009).

Characterization of adsorbents

The chemical composition of natural bentonite and rarasaponin-bentonite were determined by X-ray fluorescence method and the results are given in Table 2. The chemical composition of rarasaponin-bentonite in general is slightly different from the natural one. The change of the chemical composition of the adsorbents is possibly due to the

Table 2 The XRF analysis of natural bentonite and rarasaponinbentonite

\begin{tabular}{lcc}
\hline Compound & \multicolumn{2}{l}{ Percentage $(\%)$} \\
\cline { 2 - 3 } & Natural bentonite & Rarasaponin-bentonite \\
\hline $\mathrm{Al}_{2} \mathrm{O}_{3}$ & 15.90 & 16.30 \\
$\mathrm{SiO}_{2}$ & 49.80 & 49.10 \\
$\mathrm{Fe}_{2} \mathrm{O}_{3}$ & 7.94 & 7.72 \\
$\mathrm{CaO}$ & 2.36 & 1.83 \\
$\mathrm{~K}_{2} \mathrm{O}$ & 0.93 & 0.87 \\
$\mathrm{Na}_{2} \mathrm{O}$ & 0.08 & 0.09 \\
$\mathrm{MgO}$ & 2.41 & 2.89 \\
Other & 20.58 & 21.20 \\
\hline
\end{tabular}




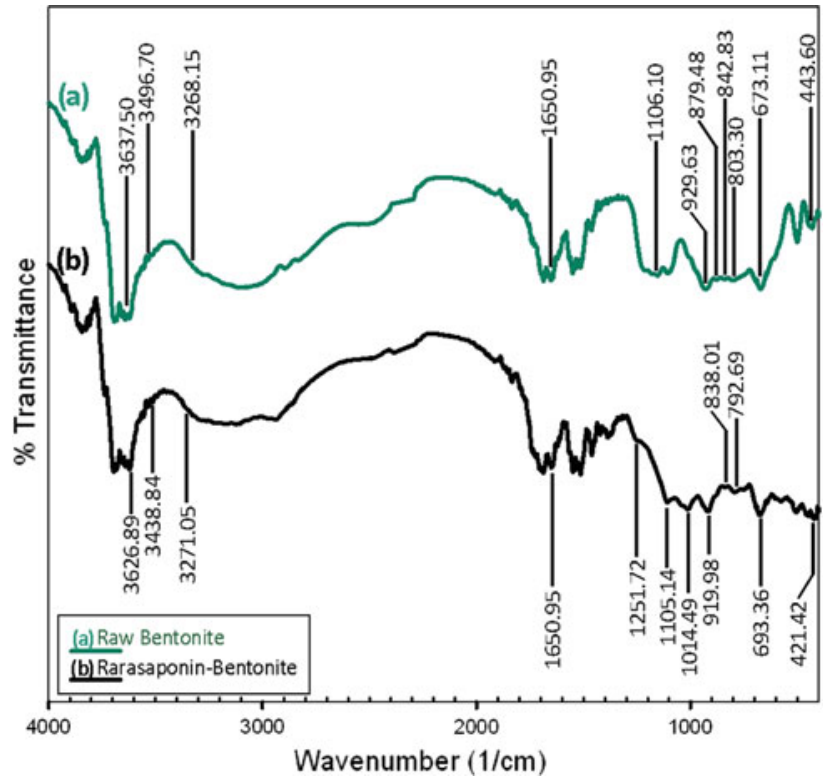

Fig. 2 FTIR spectra of natural bentonite and rarasaponin-bentonite

attachment of the functional groups from rarasaponin structure on the surface of bentonite.

The FTIR-spectra result (Fig. 2) showed that there are some functional groups either appear or lost after the modification process. The spectral bands were observed at 673.11 and $674.07 \mathrm{~cm}^{-1}$, respectively, for natural bentonite and rarasaponin-bentonite, which represent the Al-O-Si groups of the octahedral sheet, while the $\mathrm{Si}-\mathrm{O}-$ $\mathrm{Si}$ bond on tetrahedral sheet appears at $443.60 \mathrm{~cm}^{-1}$ for natural bentonite and shifting to $421.42 \mathrm{~cm}^{-1}$ for rarasaponin-bentonite. For rarasaponin-bentonite, a spectral band observed at $1,251.72 \mathrm{~cm}^{-1}$ indicates the $\mathrm{C}=\mathrm{O}$ stretch of deacylated group from rarasaponin. This evidence shows that the interaction between rarasaponin and natural bentonite occurred during modification process. For easy reference, the FTIR spectra of both adsorbents are also tabulated in Table 3.

Figure 3 depicts the XRD results of natural bentonite and rarasaponin-bentonite. The basal spacing $d_{001}$ for natural bentonite and rarasaponin-bentonite is 14.9940 and $16.6202 \AA$ at $5.8895(2 \theta)$ and $5.3128(2 \theta)$, respectively. From these data, montmorillonite was identified as major component from natural bentonite (Tabak et al. 2007). This basal spacing was expanded after modification process with rarasaponin possibly due to intercalation of rarasaponin molecules into bentonite interlayer spaces (Kurniawan et al. 2011). The expansion of natural bentonite interlamellar spacing will affect surface characteristic of rarasaponin-bentonite, including polarizability, electronic charge, and pore dimension (Do 1998).
Table 3 FTIR spectra of natural bentonite and rarasaponin-bentonite

\begin{tabular}{lcc}
\hline Functional group & \multicolumn{2}{c}{ Wavenumber $(1 / \mathrm{cm})$} \\
\cline { 2 - 3 } & $\begin{array}{l}\text { Natural } \\
\text { bentonite }\end{array}$ & $\begin{array}{l}\text { Rarasaponin- } \\
\text { bentonite }\end{array}$ \\
\hline $\mathrm{Al}(\mathrm{Mg})-\mathrm{O}-\mathrm{H}$ stretching & $3,637.50$ & $3,626.89$ \\
$\mathrm{H}-\mathrm{O}-\mathrm{H}$ stretching (for $\left.\mathrm{H}_{2} \mathrm{O}\right)$ & $3,496.70$ & $3,438.84$ \\
$\mathrm{O}-\mathrm{H}$ stretching of silanol (Si-OH) groups & $3,268.15$ & $3,271.05$ \\
$\mathrm{H}-\mathrm{O}-\mathrm{H}$ bending & $1,650.95$ & $1,650.95$ \\
$\mathrm{C}=\mathrm{O}$ stretch of deacylated carbonyl group & - & $1,251.72$ \\
$\mathrm{Si}-\mathrm{O}-\mathrm{Si}$ stretching & - & $1,014.49$ \\
$\mathrm{Si}-\mathrm{O}$ stretching & $1,106.10$ & $1,105.14$ \\
$\mathrm{O}-\mathrm{H}$ bending bounded $2 \mathrm{Al}^{3+}$ & 929.63 & 919.98 \\
$\mathrm{O}-\mathrm{H}$ bending bounded $\mathrm{Mg}^{2+}$ and $\mathrm{Al}^{3+}$ & 842.83 & 838.01 \\
$\mathrm{O}-\mathrm{H}$ bending bounded $\mathrm{Fe}^{3+}$ and $\mathrm{Al}^{3+}$ & 879.48 & - \\
$\mathrm{Si}-\mathrm{O}$ stretching of silica and quartz & 803.30 & 792.69 \\
$\mathrm{Al}-\mathrm{O}-\mathrm{Si}$ bending (for octahedral $\mathrm{Al}^{3+}$ & 673.11 & 674.07 \\
$\mathrm{Si}-\mathrm{O}-\mathrm{Si}$ bending & 443.60 & 421.42 \\
\hline
\end{tabular}

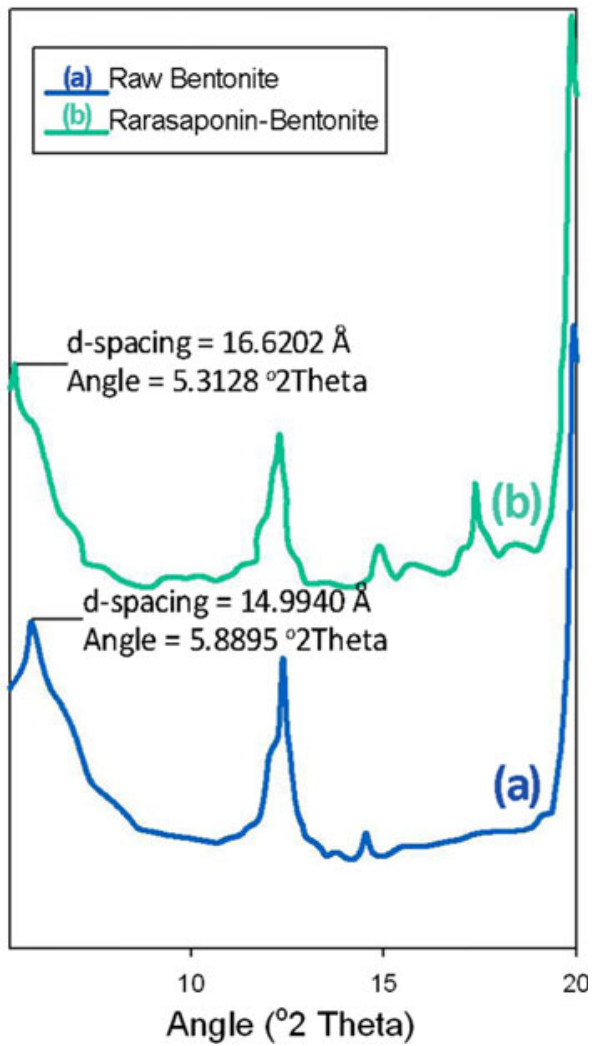

Fig. 3 XRD results of natural bentonite and rarasaponin-bentonite

Adsorption study

Several adsorption isotherm models which initially developed for gas-phase adsorption can be used to represent the liquid-phase adsorption experimental data (Rahardjo et al. 2011). In this study, four isotherm models (Langmuir, Freundlich, Sips, and Toth) with their temperature 
dependent forms were employed to correlate the adsorption experimental data of Evans blue onto natural and rarasaponin-bentonite. A non-linear least square method was employed to obtain the parameters of the adsorption models. To obtain the best fitted parameters of each model, the sum of squared error was employed as objective function to be minimized.

$\mathrm{SSE}=\left[\frac{\left(\sum q_{\mathrm{e}(\mathrm{exp})}-q_{\mathrm{e}(\mathrm{cal})}\right)^{2}}{n}\right]^{1 / 2}$

where $q_{\mathrm{e}(\exp )}(\mathrm{mmol} / \mathrm{g})$ and $q_{\mathrm{e}(\mathrm{cal})}(\mathrm{mmol} / \mathrm{g})$ are the actual amount and the calculated value of dye adsorbed by the adsorbent, respectively. While $n$ is the total number of experimental data used.

Figures 4 and 5 show the adsorption equilibrium isotherms of Evans blue on natural bentonite and rarasaponin-bentonite at various temperatures, and the fits of

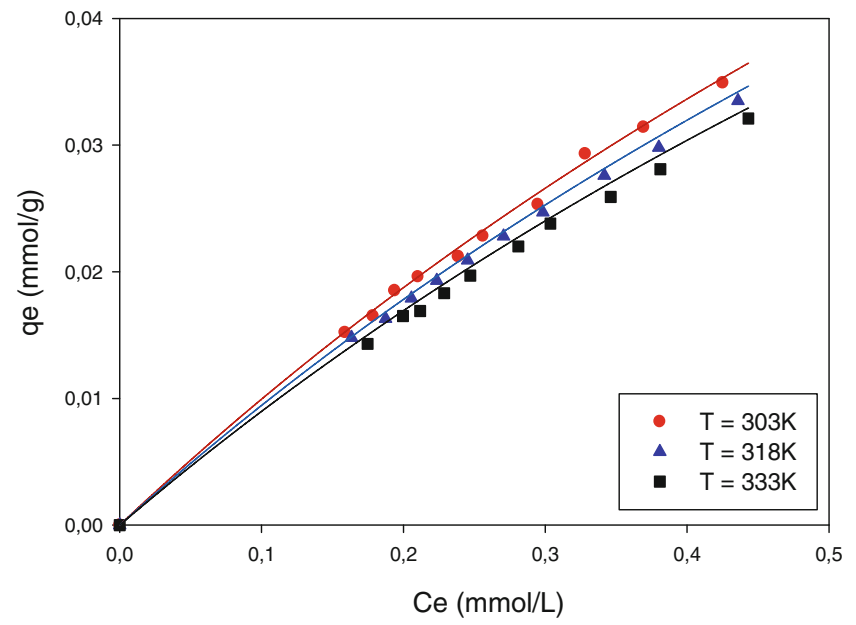

(a)

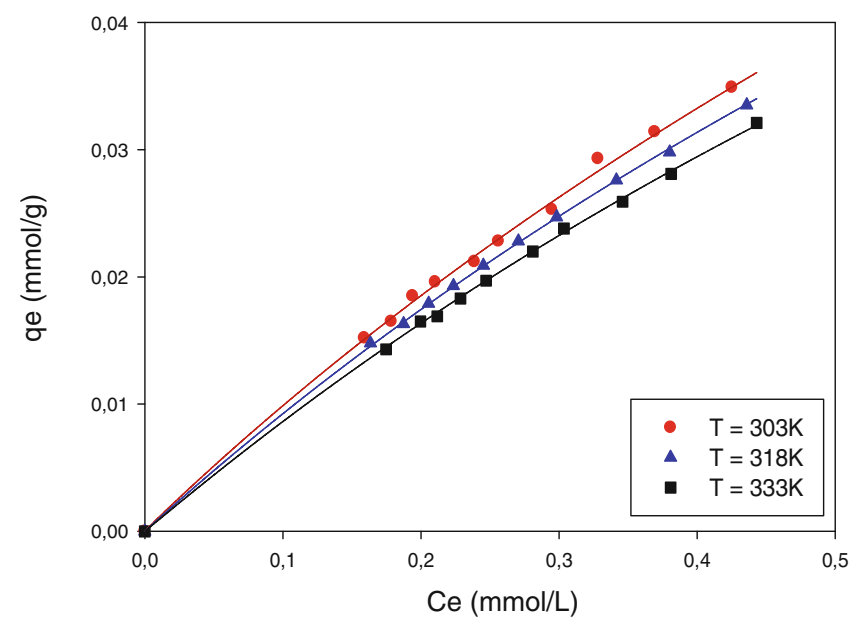

(c) different adsorption isotherm models. In these figures, the isotherm fittings are represented as solid lines while the experimental data are given as symbols. It seems that all the models can represent the adsorption experimental data well. The optimal parameters from the fitting of Langmuir, Freundlich, Sips, and Toth equations with the adsorption experimental data are summarized in Table 4.

Even visually all the models can represent the data well as seen in Figs. 4 and 5, however, the decision to choose the correct isotherm should not be based just only on how good the model represents the data visually or the value of SSE. The decision should be based on the physical meaning of each fitted parameter obtained. If the values of fitted parameters of the model are reasonable and consistent with the physical meaning of the parameter, it means that the model is applicable and can be used to represent the experimental data.

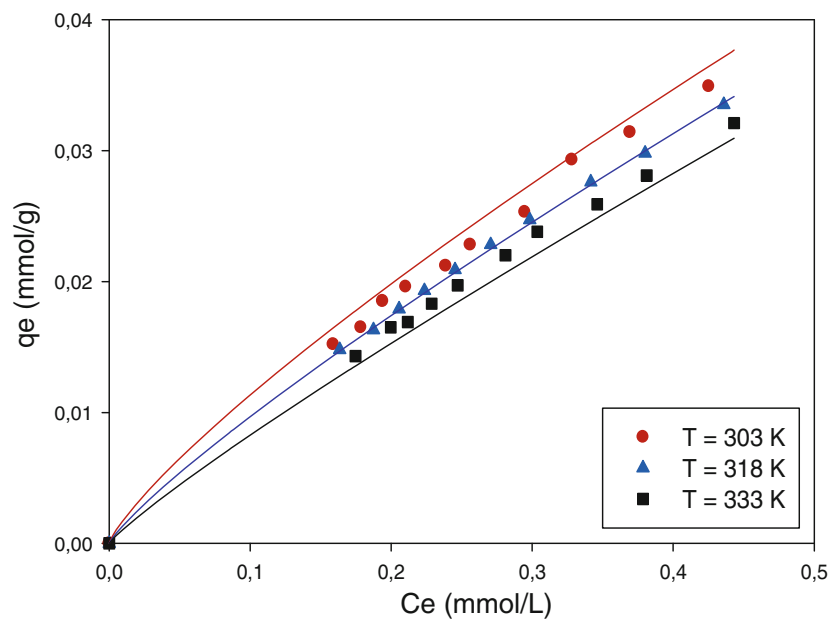

(b)

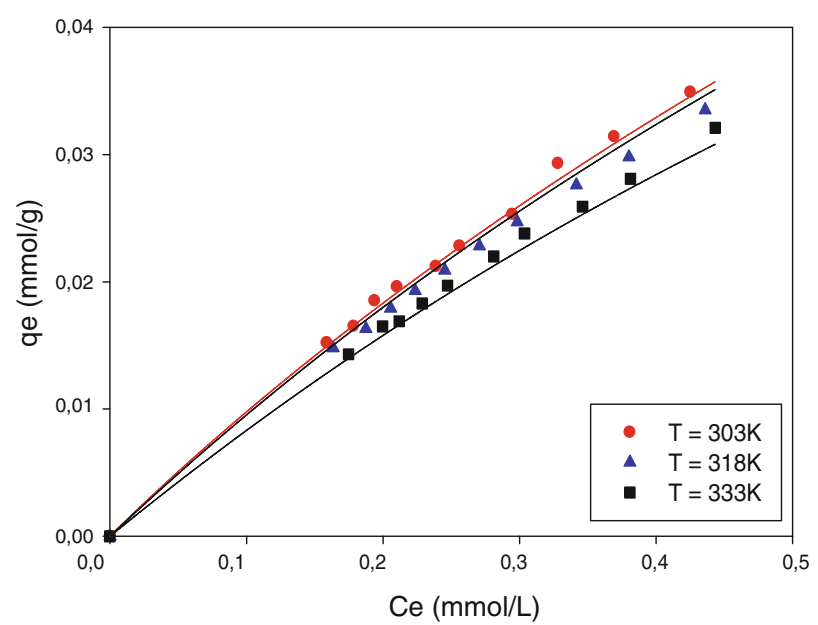

(d)

Fig. 4 Adsorption experimental data of Evans blue onto natural bentonite and the model fitted by a Langmuir, b Freundlich, c Sips, and $\mathbf{d}$ Toth isotherms 


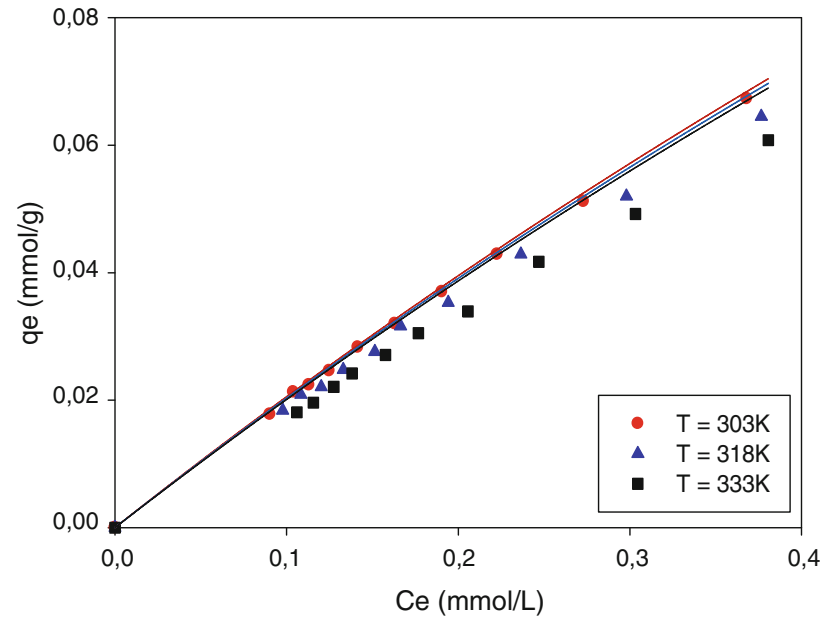

(a)

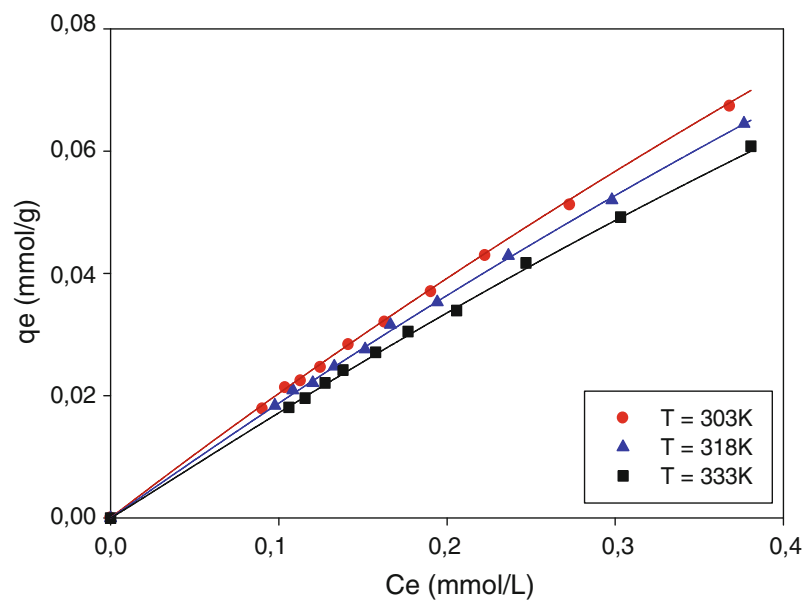

(c)

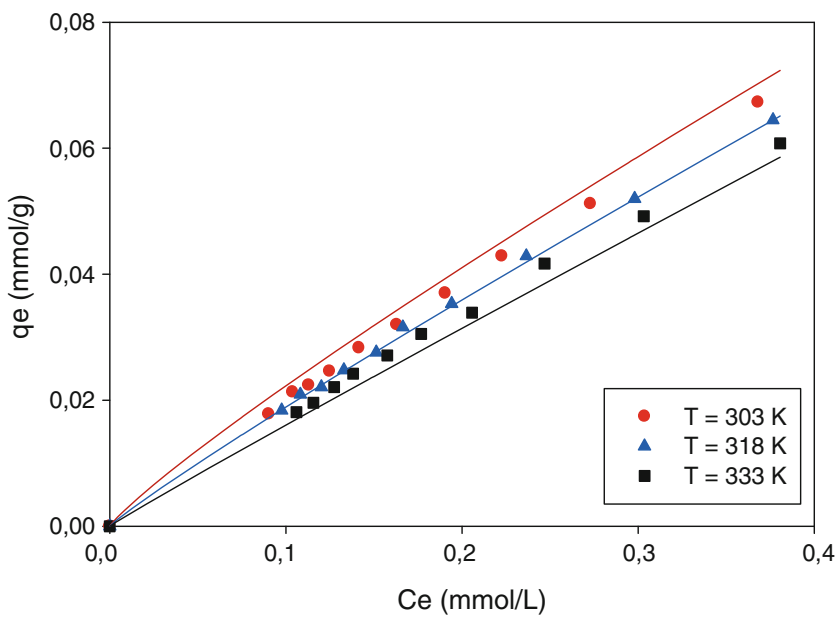

(b)

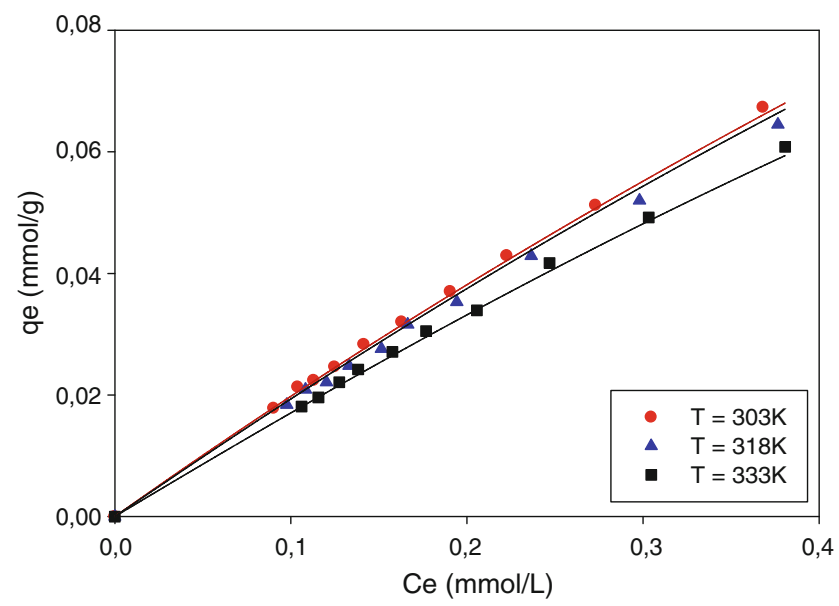

(d)

Fig. 5 Adsorption experimental data of Evans blue onto rarasaponin-bentonite and the model fitted by a Langmuir, b Freundlich, c Sips, and d Toth isotherms

Since all the models used in this study can represent the experimental data well with small values of SSE, we will discuss further about the consistency of the physical meaning of each parameters listed in Table 4. The adsorption capacity of the adsorbent is given by parameter $q_{\max }^{\mathrm{o}}$ for Langmuir, Sips and Toth, and $K_{\mathrm{F}}^{\mathrm{o}}$ for Freundlich. The parameter of the adsorption capacity for all models, both for natural bentonite and rarasaponin-bentonite, is reasonable and the value is consistent with literatures as indicated in Table 4.

The Langmuir, Sips, and Toth models have the parameter $\delta$ which represents the temperature coefficient of expansion of adsorbate. As mentioned by Ismadji and Bhatia (2001) this parameter is specific for a given component and independent of the type of adsorbent. From Table 4 it can be seen that only Toth equation gave consistent fitted parameter $\delta$. While the fitted parameter $\delta$ for
Langmuir and Sips is not consistent and dependent on the type of adsorbent. So, essentially Langmuir and Sips models fail to correlate the adsorption data of Evans blue onto natural bentonite and rarasaponin-bentonite.

As mentioned previously, $k_{\mathrm{L}}^{0}$ (Langmuir), $k_{5}^{0}$ (Sips), and $k_{\mathrm{Tk}}^{\mathrm{o}}$ (Toth) are affinity parameters. These parameter measures how strong an adsorbate molecule is attracted onto a surface. When the affinity parameter is high, the surface is covered with more adsorbate molecules as a result of stronger affinity towards the surface of adsorbent (Do 1998). Figures 4 and 5 clearly indicate that rarasaponinbentonite has higher adsorption capacity than its parent form. This is a strong indication that Evans blue has stronger adsorption affinity towards the surface of rarasaponin-bentonite than natural bentonite. Inconsistency of the fitted affinity parameter value was observed for Langmuir and Sips as seen in Table 4. Since Langmuir and Sips 
Table 4 The fitted temperature dependent parameters of several isotherm models

\begin{tabular}{|c|c|c|c|}
\hline Isotherm & Parameters & Natural bentonite & Organo-bentonite \\
\hline \multirow[t]{5}{*}{ Langmuir } & $q_{\max }^{0}(\mathrm{mmol} / \mathrm{g})$ & 0.167 & 0.527 \\
\hline & $\delta \times 10^{2}\left(\mathrm{~K}^{-1}\right)$ & 0.340 & 0.070 \\
\hline & $K_{\mathrm{L}}^{0}(\mathrm{~L} / \mathrm{mmol})$ & 0.452 & 0.114 \\
\hline & $E(\mathrm{~kJ} / \mathrm{mol})$ & 0.88 & 3.15 \\
\hline & SSE & 0.06 & 3.34 \\
\hline \multirow[t]{4}{*}{ Freundlich } & $k_{F}^{0}(\mathrm{mmol} / \mathrm{g})(\mathrm{mmol} / \mathrm{L})^{-n}$ & 0.274 & 0.612 \\
\hline & $\alpha$ & 1.646 & 1.457 \\
\hline & $A_{0}$ & 3.121 & 2.858 \\
\hline & SSE & 0.03 & 0.05 \\
\hline \multirow[t]{7}{*}{ Sips } & $q_{\max }^{0}(\mathrm{mmol} / \mathrm{g})$ & 0.185 & 0.575 \\
\hline & $\delta \times 10^{2}\left(\mathrm{~K}^{-1}\right)$ & 0.810 & 1.330 \\
\hline & $K_{\mathrm{S}}^{0}(\mathrm{~L} / \mathrm{mmol})$ & 0.542 & 0.367 \\
\hline & $E(\mathrm{~kJ} / \mathrm{mol})$ & 5.29 & 9.64 \\
\hline & $n_{0}$ & 1.021 & 1.008 \\
\hline & $\eta$ & 0.299 & 0.405 \\
\hline & SSE & 0.03 & 0.05 \\
\hline \multirow[t]{7}{*}{ Toth } & $q_{\max }^{0}(\mathrm{mmol} / \mathrm{g})$ & 0.263 & 0.516 \\
\hline & $\delta \times 10^{2}\left(\mathrm{~K}^{-1}\right)$ & 2.380 & 2.230 \\
\hline & $K_{\mathrm{Th}}^{0}(\mathrm{~L} / \mathrm{mmol})$ & 2.051 & 2.524 \\
\hline & $E(\mathrm{~kJ} / \mathrm{mol})$ & 9.63 & 11.43 \\
\hline & $t_{0}$ & 0.779 & 0.978 \\
\hline & $\eta$ & 3.383 & 4.243 \\
\hline & SSE & 0.07 & 0.09 \\
\hline
\end{tabular}

models fail to give reasonable and consistent parameters (temperature coefficient of expansion of adsorbate and adsorption affinity), both of these models will not be included in subsequent discussion.

The heterogeneity of the system is given by parameter $t_{0}$ for Toth equation and parameter $A_{0}$ for Freundlich model. As mentioned by Do (1998) that the system heterogeneity could stem from the solid or the adsorbate or a combination of both. During the modification process, the acyl $\left(\mathrm{C}_{2} \mathrm{H}_{3} \mathrm{O}^{+}\right)$groups which are attached to the carbonyl group of the rarasaponin molecules were deacylated and attached to the protonated silanol groups which are available in tetrahedral sheet of natural bentonite (Kurniawan et al. 2011), the attachment of the rarasaponin molecules into interlayer structure of natural bentonite increases the heterogeneity of the system. This phenomenon was not captured by parameter $A_{0}$ in Freundlich model, the value of this parameter decreases with increase of the system heterogeneity. The value of parameter $t_{0}$ in Toth equation increases with increase of the system heterogeneity as indicated in Table 4. Therefore, only Toth model still can represent the adsorption experimental data. In order to determine the applicability of Toth equation to represent the adsorption experimental data of Evans blue onto natural bentonite and rarasaponin-bentonite we still need to examine the rest of fitted parameters $(E$ and $\eta)$.

In the physical adsorption, the temperature has been known to have negative effect on the amount of adsorbate adsorbed by the solid. The uptake of adsorbate decreases with increase of temperature. Physical adsorption processes usually have isosteric heat of adsorption $<40 \mathrm{~kJ} / \mathrm{mol}$ (Do 1998). Figures 4 and 5 show that the adsorption of Evans blue onto natural bentonite and rarasaponin-bentonite is mainly controlled by physical adsorption. In the physical adsorption, the increase of temperature weakens the interaction between Evans blue and natural bentonite or rarasaponin-bentonite, therefore the amount of dye uptake by both of the adsorbents decreased with increasing temperature. The results of parameter $E$ from the fitting of Toth equation is consistent with our experimental data as depicted in Figs. 4 and 5. Higher rate of heat adsorption on rarasaponin-bentonite is an indication that other bonding mechanism also took place during the process. As mentioned in the previous paragraph that the acyl groups in the rarasaponin structure play significant role during the formation of rarasaponin-bentonite. Some of these acyl groups were deacylated during the formation of rarasaponin-bentonite and some of them remain in the carbonyl group of 

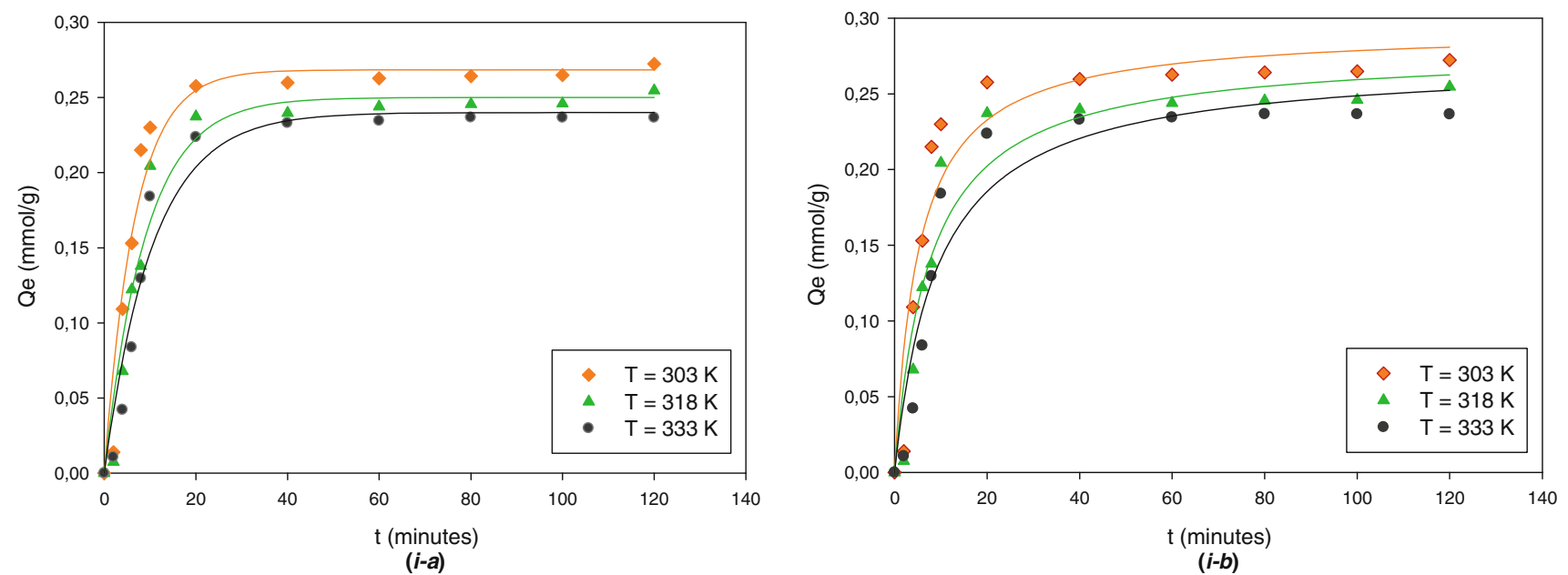

$(i-b)$
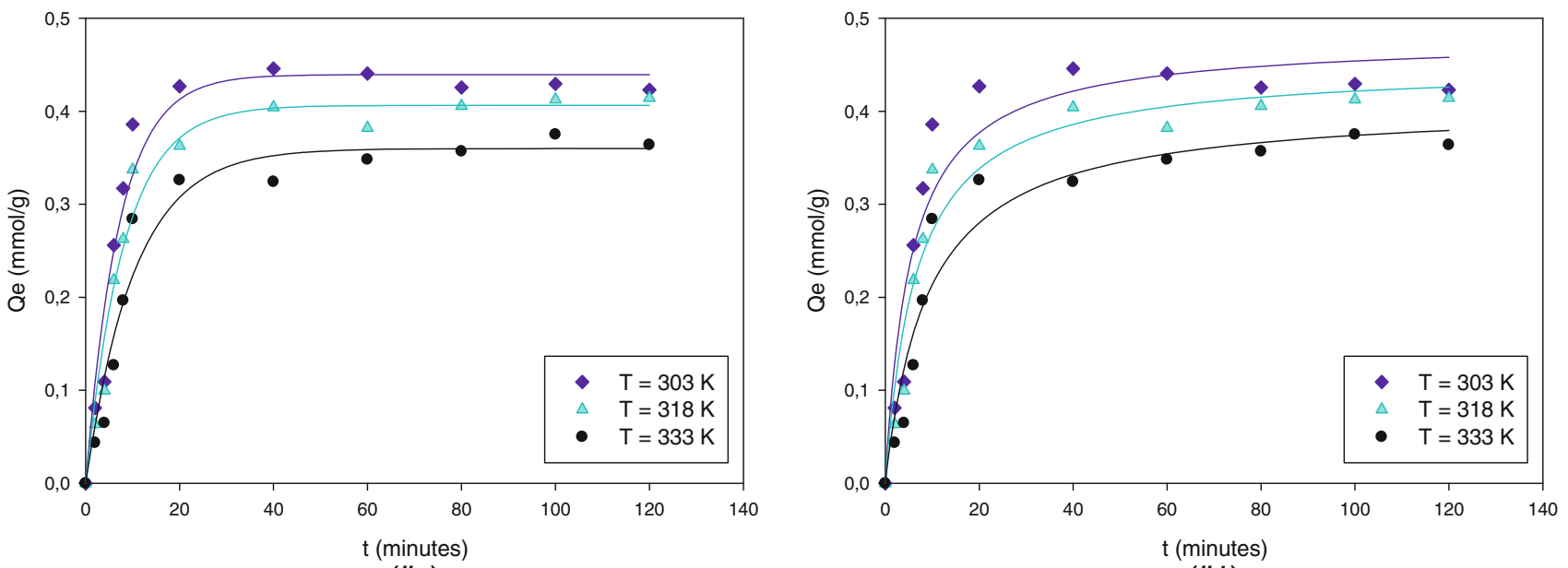

(ii-a)

$(i i-b)$

Fig. 6 Kinetics experimental data of Evans blue onto natural bentonite and fitted model by (i-a) pseudo-first order, (i-b) pseudo-second order models, and using rarasaponin-bentonite (ii-a) pseudo-first order, (ii-b) pseudo-second order models

Table 5 kinetic parameters for pseudo-first and pseudo-second order models

\begin{tabular}{|c|c|c|c|c|c|c|c|c|}
\hline \multirow[t]{2}{*}{$\mathrm{T}(\mathrm{K})$} & \multicolumn{4}{|c|}{ Pseudo-first order } & \multicolumn{4}{|l|}{ Pseudo-second order } \\
\hline & $k_{1}\left(\min ^{-1}\right)$ & $q_{\mathrm{e}}(\mathrm{mmol} / \mathrm{g})$ & $R^{2}$ & SSE & $k_{2}\left(\mathrm{~g} \cdot \mathrm{mmol}^{-1} \mathrm{~min}^{-1}\right)$ & $q_{\mathrm{e}}(\mathrm{mmol} / \mathrm{g})$ & $R^{2}$ & SSE \\
\hline \multicolumn{9}{|c|}{ Using natural bentonite as adsorbent } \\
\hline 303 & 0.1491 & 0.2683 & 0.9578 & 0.0215 & 0.6593 & 0.2929 & 0.9204 & 0.0295 \\
\hline 318 & 0.1107 & 0.2499 & 0.9609 & 0.0201 & 0.4721 & 0.2791 & 0.9316 & 0.0266 \\
\hline 333 & 0.0945 & 0.2399 & 0.9574 & 0.0208 & 0.3946 & 0.2719 & 0.9267 & 0.0272 \\
\hline \multicolumn{9}{|c|}{ Using rarasaponin-bentonite as adsorbent } \\
\hline 303 & 0.1403 & 0.4393 & 0.9607 & 0.0336 & 0.3873 & 0.4785 & 0.9188 & 0.0483 \\
\hline 318 & 0.1223 & 0.4063 & 0.9704 & 0.0272 & 0.3379 & 0.4494 & 0.9474 & 0.0362 \\
\hline 333 & 0.0964 & 0.3599 & 0.9575 & 0.0303 & 0.2678 & 0.4082 & 0.9388 & 0.0364 \\
\hline
\end{tabular}

rarasaponin structure. In polar solutions such as water, excess acyl groups in the surface of rarasaponin-bentonite became positively charged. On the other side, the Evans blue which belongs to Azo dye category was negatively charged due to its $\mathrm{SO}_{3}$ functional groups. The electrostatic interaction between positive charge of acyl groups and 
negative charge of Evans blue occurred, leading to higher rate of heat adsorption.

In the Toth equation, parameter $\eta$ also measures the heterogeneity of the system. If the value of this parameter deviates from unity, the system is more heterogeneous. The value of fitted parameter $\eta$ of Toth model increases with system heterogeneity as shown in Table 4 . Based on the evaluation of the physical meaning of fitted parameters of each equation, it is clear that the Toth equation can represent the adsorption data better than the other equations.

\section{Adsorption kinetics}

One of the most crucial factors for designing the adsorption system is the ability to predict the rate at which the adsorbate removal takes place in a given solid/solution system (Plazinski et al. 2009). Numerous kinetic models have been developed to predict the behavior of the adsorption kinetic experimental data, and most of the models were developed based on certain fundamental approach to interfacial kinetics (Plazinski et al. 2009). Most widely used models such as pseudo-first order (Lagergren 1898) and pseudo-second order (Blanchard et al. 1984) were also developed based on the interfacial kinetics approach.

Figure 6 presents the adsorption kinetic experimental data Evans blue and the calculated values using pseudofirst order and pseudo-second order models for Natural bentonite and rarasaponin-bentonite, respectively. The fitted parameters' value of pseudo-first order and pseudosecond order are summarized in Table 5. Both of the models can represent the experimental data well. The pseudo-first order gave a smaller SSE a slightly better coefficient of correlation $\left(R^{2}\right)$. The deviation of $q_{\mathrm{e}}$ obtained from the fitting and experimental data in the pseudo first order is smaller than the pseudo-second order. Based on this evidence, the controlling mechanism of the adsorption of Evans blue onto natural bentonite and rarasaponinbentonite was physical adsorption

\section{Conclusion}

The adsorption of Evans blue onto natural bentonite and rarasaponin-bentonite was studied. The modification of natural bentonite using natural surfactant (rarasaponin) increased the adsorption capacity of the bentonite. Four adsorption isotherm models with their temperature dependent forms were used to correlate the adsorption experimental data, and it was found that the Toth model gave the consistent and reasonable values of fitted parameters. For the kinetic study, the pseudo-first order gave smaller deviation of the $q_{\mathrm{e}}$ value obtained from the fitting with the experimental data. The controlling mechanism of the adsorption of Evans blue onto natural bentonite and rarasaponin-bentonite was physical adsorption, other mechanism such as electrostatic interaction also occurred. The best way to determine the adsorption mechanism is using direct spectroscopic measurement such as calorimeter, etc.

Acknowledgments The first author would like to express their sincere gratitude to the Department of Chemical Engineering National Taiwan University of Science and Technology, Taiwan, for undergraduate exchange student grant and providing all the research facilities.

\section{References}

Arief VO, Trilestari K, Sunarso J, Indraswati N, Ismadji S (2008) Recent progress on biosorption of heavy metals from liquids using low cost biosorbents: characterization, biosorption parameters and mechanism studies. Clean Soil Air Water 36:937-962

Asao Y, Morikawa T, Xie Y, Okamoto M, Hamao M, Matsuda H, Muraoka O, Yuan D, Yoshikawa M (2009) Structures of acetylated oleanane-type triterpene saponins, rarasaponins IV, $\mathrm{V}$, and VI, and anti-hyperlipidemic constituents from the pericarps of Sapindus rarak. Chem Pharm Bull 57:198-203

Blanchard G, Maunaye M, Martin G (1984) Removal of heavy metals from waters by means of natural zeolites. Water Res 18(12):15011507

Do DD (1998) Adsorption analysis : equilibria and kinetics. Imperial College Press, Australia

Febrianto J, Kosasih AN, Sunarso J, Ju YH, Indraswati N, Ismadji S (2009) Equilibrium and kinetic studies in adsorption of heavy metals using biosorbent: a summary of recent studies. J Hazard Mater 162:616-645

Ho YS (1995) Adsorption of heavy metals from waste streams by peat. PhD Thesis, The University of Birmingham, Birmingham

Ismadji S, Bhatia SK (2001) A modified pore filling isotherm for liquid phase adsorption in activated carbon. Langmuir 17:14881498

Juang RS, Lin S-H, Tsao K-H (2002) Mechanism of sorption of phenols from aqueous solutions onto surfactant-modified montmorillonite. J Colloid Interface Sci 254:234-241

Khenifi A, Bouberka Z, Sekrane F, Kameche M, Derriche Z (2007) Adsorption study of an industrial dye by an organic clay. Adsorption 13:149-158

Koswojo E, Utomo RP, Ju YH, Ayucitra A, Soetaredjo FE, Sunarso J, Ismadji S (2010) Acid green 25 removal from wastewater by organo-bentonite from Pacitan. Appl Clay Sci 48:81-86

Kurniawan A, Sutiono H, Ju YH, Soetaredjo FE, Ayucitra A, Yudha A, Ismadji S (2011) Utilization of rarasaponin natural surfactant for organo-bentonite preparation: application for methylene blue removal from aqueous effluent. Microporous Mesoporous Mater 142:184-193

Lagergren S (1898) Zur theorie der sogenannten adsorption gelöster stoffe Kungliga Svenska Vetenskapsakademiens Handlingar 24:1-39

Lee JJ, Choi J, Park JW (2002) Simultaneous sorption of lead and chlorobenzene by organobentonite. Chemosphere 49:1309-1315

Lesmana SO, Febriana N, Soetaredjo FE, Sunarso J, Ismadji S (2009) Studies on potential applications of biomass for the separation of heavy metals from water and wastewater. Biochem Eng J 44:19-41

Plazinski W, Rudzinski W, Plazinska A (2009) Theoritical models of sorption kinetics including a surface reaction mechanism: a review. Adv Colloid Interface Sci 152:2-13 
Rahardjo AK, Susanto MJJ, Kurniawan A, Indraswati N, Ismadji S (2011) Modified Ponorogo bentonite for the removal of ampicillin from wastewater. J Hazard Mater 190:1001-1008

Tabak A, Afsin B, Aygun S, F Koksal E (2007) Structural characteristics of organo-modified bentonites of diferrent origin. J Thermal Anal Calor 87:375-381
Volesky B (2007) Biosorption and me. Water Res 41:4017-4029

Wang J, Chen C (2006) Biosorption of heavy metals by Saccharomyces cerevisiae: a review. Biotechnol Adv 24:427-451

Zee FP (2002) Anaerobic azo dyes reduction. Doctoral Thesis, Wageningen University, Wageningen, The Netherlands 